\title{
A convergent and precise finite element scheme for Landau-Lifschitz-Gilbert equation
}

\author{
François Alouges · Evaggelos Kritsikis • \\ Jutta Steiner • Jean-Christophe Toussaint
}

Received: 16 November 2012 / Revised: 8 November 2013 / Published online: 25 February 2014

(C) Springer-Verlag Berlin Heidelberg 2014

\begin{abstract}
In this paper, we propose a new scheme for the numerical integration of the Landau-Lifschitz-Gilbert (LLG) equations in their full complexity, in particular including stray-field interactions. The scheme is consistent up to order 2 (in time), and unconditionally stable. It combines a linear inner iteration with a non-linear renormalization stage for which a rigorous proof of convergence of the numerical solution toward a weak solution is given, when both space and time stepsizes tend to 0 . A numerical implementation of the scheme shows its performance on physically relevant test cases. We point out that to the knowledge of the authors this is the first finite element scheme for the LLG equations which enjoys such theoretical and practical properties.
\end{abstract}

Mathematics Subject Classification $35 \mathrm{~K} 55 \cdot 65 \mathrm{M} 12 \cdot 65 \mathrm{M} 60$

\section{Introduction}

In 1935 Landau and Lifschitz proposed a model for the description of the evolution of the magnetization in a ferromagnetic material [15]: Suppose that the three dimensional ferromagnetic sample under consideration occupies some domain $\Omega \subset \mathbb{R}^{3}$ and let

\footnotetext{
F. Alouges $(\varangle)$

CMAP-École Polytechnique, Route de Saclay, 91128 Palaiseau cedex, France

e-mail: francois.alouges@polytechnique.edu

E. Kritsikis · J-C. Toussaint

Institut Néel, 25 avenue des Martyrs, Bâtiment K, BP 166, 38042 Grenoble cedex 9, France

E. Kritsikis - J-C. Toussaint

Grenoble-INP, 46 Avenue Félix Viallet, 38031 Grenoble Cedex 1, France

J. Steiner

Institute for Applied Mathematics, Mathematik Zentrum, Endenicher Allee 60, 53115 Bonn, Germany
} 
m be the direction of the magnetization. The model, namely the Landau-LifschitzGilbert (LLG) equations, reads as follows

$$
\left[\begin{array}{l}
\partial_{t} \mathbf{m}-\alpha \mathbf{m} \times \partial_{t} \mathbf{m}=-\gamma_{0} M_{s} \mathbf{m} \times \mathbf{H}_{\mathrm{eff}} \quad \text { in } \Omega \\
\partial_{n} \mathbf{m}=0 \text { on } \partial \Omega .
\end{array}\right.
$$

(As above, vectorial quantities will be denoted in bold letters in the sequel to avoid ambiguity). The parameters in the equation are the damping parameter $\alpha$, the gyromagnetic constant $\gamma_{0}$ and the saturation magnetization $M_{s}$. The so-called effective field $\mathbf{H}_{\mathrm{eff}}$, measured in units of $M_{s}$, is given by the functional derivative of the micromagnetic (free) energy $\mathcal{E}$, more precisely

$$
\mathbf{H}_{\mathrm{eff}}(\mathbf{m})=-\frac{\partial \mathcal{E}}{\partial \mathbf{m}}=d^{2} \Delta \mathbf{m}+\mathbf{H}_{\mathrm{d}}(\mathbf{m})+\mathbf{H}_{\mathrm{ext}}+Q(\mathbf{e} \cdot \mathbf{m}) \mathbf{e}
$$

where the energy $\mathcal{E}$ (see $[10,13,15])$ is given by

$$
\begin{aligned}
\mathcal{E}(\mathbf{m})= & \frac{1}{2}\left(d^{2} \int_{\Omega}|\nabla \mathbf{m}|^{2} \mathrm{~d} \mathbf{x}-\int_{\Omega} \mathbf{H}_{\mathrm{d}}(\mathbf{m}) \cdot \mathbf{m} \mathrm{d} \mathbf{x}\right. \\
& \left.-2 \int_{\Omega} \mathbf{H}_{\mathrm{ext}} \cdot \mathbf{m} \mathrm{d} \mathbf{x}-Q \int_{\Omega}(\mathbf{e} \cdot \mathbf{m})^{2} \mathrm{~d} \mathbf{x}\right) .
\end{aligned}
$$

The four contributions to the effective field in (2) and the energy in (3), respectively, correspond to the so-called exchange, stray-field, applied and anisotropy field or energy, respectively. The material constants in (2) and (3) are the exchange constant $d$, the anisotropy constant $Q$ and the anisotropy direction e (also called the easy axis). Furthermore, the vector field $\mathbf{H}_{\text {ext }}$ models an applied magnetic field. We will also use the notation $\mathbf{H}_{\text {aniso }}=Q(\mathbf{e} \cdot \mathbf{m})$ e. The stray field $\mathbf{H}_{\mathrm{d}}(\mathbf{m})$ is the magnetic field induced by the magnetization distribution $\mathbf{m}$ via the following (subset of) static Maxwell equations

$$
\left[\begin{array}{l}
\operatorname{curl} \mathbf{H}_{\mathrm{d}}(\mathbf{m})=0 \quad \text { in } \mathbb{R}^{3} \\
\operatorname{div}\left(\mathbf{H}_{\mathrm{d}}(\mathbf{m})+\mathbf{m}\right)=0 \quad \text { in } \mathbb{R}^{3}
\end{array}\right.
$$

Notice that in the equation above, all fields are measured in units of $M_{s}$. Below the Curie temperature, the magnetization can be described by a directional field that we rescale to unit length. It is straightforward to check that the magnitude of the magnetization

$$
|\mathbf{m}(\mathbf{x}, t)|=1
$$

is formally conserved by the dynamics (1). Take note that the gyromagnetic term is a conservative term while the damping term leads to the following energy dissipation law 


$$
\frac{\mathrm{d}}{\mathrm{d} t} \mathcal{E}(\mathbf{m}(t))=-\frac{\alpha}{\gamma_{0} M_{s}} \int_{\Omega}\left|\partial_{t} \mathbf{m}\right|^{2} \mathrm{~d} \mathbf{x} .
$$

In the mathematical analysis, most of the physical constants can be normalized to one by rescaling. For example, rescaling time and redefining $\alpha$ allows to assume that $\gamma_{0} M_{s}=1$. However, in the last section of this paper, where the proposed numerical scheme is tested in physical situations, it is more appropriate to go back to the original scaling.

The numerical approximation of solutions to (1) is an important issue in applications. Nowadays, numerous strategies exist in the literature-though among them only few reliable ones [11]. Classical schemes are based on finite differences that are, as usual, well adapted to Cartesian grids. On the other hand, finite element approximations will suit complex geometries and weak solutions, with the drawback that they are difficult to analyze in practice. In particular, proving the convergence of a finite element solution towards a solution of (1) as the space and time steps tend to zero turns out to be quite difficult and has probably been first established in [5]. This result was further improved in [8] and [1] for the case in which only the exchange term is present, and in [3] for the general case. Such formulations are now in use in the physics literature [16]. We hereafter study a further generalization of the scheme proposed in [1]: a second order (in time) variant. We point out that despite the fact that LLG equation is highly non-linear, our scheme still uses an inner iteration which requires a linear and positive definite system to be solved. Numerical tests support the performance of the method. Notice also that convergent order 2 schemes, based for instance on midpoint approximations have previously been proposed in [9] with the drawback of needing to solve a non-linear inner iteration.

Let us start with a brief outline of our paper. In Sect. 2 we first recall the notion of weak solutions. Section 3 introduces the finite-element spaces. Section 4 restates the first order scheme proposed in [1]. The non-linearity of the LLG equation calls for recurrent renormalization of the time-discrete approximation, an issue discussed in Sect. 5. Section 6 finally provides a derivation of our new scheme, the main convergence result together with the proof. Section 7 finally covers some numerical examples.

\section{Notion of weak solutions to LLG}

Let us recall the notion of a weak solution to (1) from [6] and [18].

Definition 1 Consider an initial magnetization, i.e., a vector field $\mathbf{m}_{0} \in H^{1}(\Omega)^{3}$ that is a.e. of unit length. A vector field $\mathbf{m}$ is called a weak solution to (1) with initial data $\mathbf{m}_{0}$ if for all times $T>0$ there holds

1. $\mathbf{m} \in H^{1}\left(\Omega_{T}\right)^{3}$ with $\Omega_{T}=\Omega \times(0, T)$, and $|\mathbf{m}|=1$ a.e.

2. For all test functions $\in \mathbf{H}^{\mathbf{1}}\left(\Omega_{\mathbf{T}}\right)^{\mathbf{3}}$ 


$$
\begin{aligned}
& \int_{\Omega_{T}} \partial_{t} \mathbf{m} \cdot \Psi \mathrm{d} \mathbf{x} \mathrm{d} t-\alpha \int_{\Omega_{T}}\left(\mathbf{m} \times \partial_{t} \mathbf{m}\right) \cdot \Psi \mathrm{d} \mathbf{x} \mathrm{d} t \\
& \quad=d^{2} \sum_{i=1}^{d} \int_{\Omega_{T}}\left(\mathbf{m} \times \partial_{x_{i}} \mathbf{m}\right) \cdot \partial_{x_{i}} \Psi \mathrm{d} \mathbf{x} \mathrm{d} t \\
& \quad-\int_{\Omega_{T}} \mathbf{m} \times\left(\mathbf{H}_{\mathrm{d}}(\mathbf{m})+\mathbf{H}_{\mathrm{ext}}+\mathbf{H}_{\mathrm{aniso}}(\mathbf{m})\right) \cdot \Psi \mathrm{d} \mathbf{x} \mathrm{d} t
\end{aligned}
$$

3. The magnetization initially satisfies $\mathbf{m}(\mathbf{x}, 0)=\mathbf{m}_{0}(\mathbf{x})$ in the trace sense, and

4. The energy decreases according to

$$
\mathcal{E}(\mathbf{m}(T))+\alpha \int_{\Omega_{T}}\left|\partial_{t} \mathbf{m}\right|^{2} \mathrm{~d} \mathbf{x} \mathrm{d} t \leq \mathcal{E}(\mathbf{m}(0)) .
$$

\section{The finite element scheme}

As in [5], our discretization relies on piecewise linear finite elements in space combined with a linear interpolation in time. The domain $\Omega$ is discretized by a conformal triangulation $\mathcal{T}_{h}$ of mesh size $h$ with vertices $\left(\mathbf{x}_{i}^{h}\right)_{1 \leq i \leq N_{h}}$. Let us denote by $\left(\phi_{i}^{h}\right)_{1 \leq i \leq N_{h}}$ the set of associated piecewise linear basis functions that satisfy $\phi_{i}^{h}\left(\mathbf{x}_{j}^{h}\right)=\delta_{i, j}$ at the vertices $\mathbf{x}_{j}^{h}$ for $1 \leq i, j \leq N_{h}$, where $\delta_{i, j}$ denotes the Kronecker symbol. This amounts to a standard $P^{1}\left(\mathcal{T}_{h}\right)$-discretization. Based on the scalar basis $\left(\phi_{i}^{h}\right)_{1 \leq i \leq N_{h}}$ we construct the vector-valued finite element space as

$$
V_{h}=\left\{\mathbf{u}^{h}=\sum_{i} \mathbf{u}_{i} \phi_{i}^{h}, \text { s.t. } \forall i, \mathbf{u}_{i} \in \mathbb{R}^{3}\right\} .
$$

Due to the unit-length constraint (5), the solution to (7) is sought in the subset

$$
M_{h}=\left\{\mathbf{u}^{h} \in V_{h}, \text { s.t. } \forall i, \mathbf{u}_{i} \in \mathbb{S}^{2}\right\} \subset V_{h} .
$$

Let us also introduce the tangent space to $\mathbf{m}^{h}=\sum_{i} \mathbf{m}_{i} \phi_{i}^{h} \in M_{h}$, denoted by

$$
K_{\mathbf{m}}=\left\{\mathbf{v}^{h}=\sum_{i} \mathbf{v}_{i} \phi_{i}^{h}, \text { s.t. } \forall i, \mathbf{v}_{i} \cdot \mathbf{m}_{i}=0\right\} .
$$

Furthermore, the classical nodal interpolation operator is given by

$$
\begin{aligned}
\mathcal{I}_{h}: \mathcal{C}^{0}\left(\Omega, \mathbb{R}^{3}\right) & \rightarrow V_{h} \\
\mathbf{u} & \mapsto \sum_{i} \mathbf{u}\left(\mathbf{x}_{i}^{h}\right) \phi_{i}^{h} .
\end{aligned}
$$


To simplify notations, the index $h$ of the ansatz functions will be neglected from now on most of the times, i.e., we write $\mathbf{u}, \mathbf{v}$, etc. instead of $\mathbf{u}^{h}, \mathbf{v}^{h}$, respectively, in case this does not lead to any ambiguities.

\section{Revisiting the $\theta$-scheme}

The finite element scheme proposed in [5] relies on the observation that the LLG Eq. (1) — with the notation $\mathbf{v}=\partial_{t} \mathbf{m}$-can be rewritten in the following weak form

$$
\begin{aligned}
& \alpha \int_{\Omega} \mathbf{v} \cdot \Psi \mathrm{d} \mathbf{x}+\alpha \int_{\Omega} \mathbf{m} \times \mathbf{v} \cdot \Psi \mathrm{d} \mathbf{x} \\
& \quad=-d^{2} \int_{\Omega} \nabla \mathbf{m} \cdot \nabla \Psi \mathrm{d} \mathbf{x}+\int_{\Omega}\left(\mathbf{H}_{\mathrm{d}}(\mathbf{m})+\mathbf{H}_{\mathrm{ext}}+\mathbf{H}_{\text {aniso }}(\mathbf{m})\right) \cdot \Psi \mathrm{d} \mathbf{x} .
\end{aligned}
$$

Equation (10) holds for every test function $\Psi \in H^{1}\left(\Omega, \mathbb{R}^{3}\right)$ that satisfies $\Psi(\mathbf{x}) \cdot \mathbf{m}(\mathbf{x})=$ 0 for a.e. $\mathbf{x}$ in $\Omega$. The reformulation of (1) in the form of (10) motivated the following $\theta$-scheme introduced in [1]:

Algorithm 1 Given an initial $\mathbf{m}^{0} \in M_{h}$ choose $\theta \in[0,1]$ and a time step size $k=\frac{T}{N}$ with $N \in \mathbb{N}$. For $n=0,1, \ldots, N$

$$
\left[\begin{array}{l}
\text { a) Find } \mathbf{v}^{n} \in K_{\mathbf{m}^{n}} \text { such that for all test functions } \Psi \in K_{\mathbf{m}^{n}} \\
\alpha \int_{\Omega} \mathbf{v}^{n} \cdot \Psi \mathrm{d} \mathbf{x}+\int_{\Omega} \mathbf{m}^{n} \times \mathbf{v}^{n} \cdot \Psi \mathrm{d} \mathbf{x}=-d^{2} \nabla\left(\mathbf{m}^{n}+\theta k \mathbf{v}^{n}\right) \cdot \nabla \Psi \mathrm{d} \mathbf{x} \\
+\int_{\Omega}\left(\mathbf{H}_{\mathrm{d}}\left(\mathbf{m}^{n}\right)+\mathbf{H}_{\mathrm{ext}}+\mathbf{H}_{\text {aniso }}\left(\mathbf{m}^{n}\right)\right) \cdot \Psi \mathrm{d} \mathbf{x} \\
\text { b) Set } \mathbf{m}^{n+1}=\sum_{i} \mathbf{m}_{i}^{n+1} \phi_{i}^{h}, \text { where } \forall i, \mathbf{m}_{i}^{n+1}=\frac{\mathbf{m}_{i}^{n}+k \mathbf{v}_{i}^{n}}{\left|\mathbf{m}_{i}^{n}+k \mathbf{v}_{i}^{n}\right|} .
\end{array}\right.
$$

It is noteworthy that this procedure requires the solution of a linear equation in each time step only. Moreover, due to the fact that the symmetric part of the underlying matrix is positive definite, existence and uniqueness of a solution to (11) is guaranteed.

The time discrete solution constructed via algorithm (11) at time-steps $N=\left[\frac{T}{k}\right]$ is interpolated as follows:

Definition 2 In each time interval $t \in[n k,(n+1) k)$ with $n \in\{0, \cdots, N\}$ we set

$$
\begin{aligned}
\mathbf{m}_{h, k}(\mathbf{x}, t) & =\frac{t-n k}{k} \mathbf{m}^{n+1}(\mathbf{x})+\frac{(n+1) k-t}{k} \mathbf{m}^{n}(\mathbf{x}), \\
\mathbf{m}_{h, k}^{-} & =\mathbf{m}^{n}(\mathbf{x}), \quad \mathbf{v}_{h, k}=\mathbf{v}^{n}(\mathbf{x}) .
\end{aligned}
$$


Our notational convention is thus that $\mathbf{m}_{h, k}, \mathbf{m}_{h, k}^{-}$and $\mathbf{v}_{h, k}$ refer to suitable time interpolants of the time discrete approximation $\mathbf{m}^{n}$ and $\mathbf{v}^{n}$. Notice that $\mathbf{m}_{h, k}$ is piecewise linear in time whereas $\mathbf{m}_{h, k}^{-}$and $\mathbf{v}_{h, k}$ are piecewise constant. (The introduction of the piecewise constant magnetization will be useful in the convergence proof). Based on this discretization, weak convergence of the constructed approximation was established in [1]. Both the proof of this result and the proof in case of our new scheme consist of the following two main "classical" steps: as a first step establishing an energy estimate which guarantees the convergence (sufficiently strong) of the sequence constructed and then in a second step verifying that the limit indeed satisfies the equation. As far as the first step is concerned, the following section addresses the fact that the energy behaves well under renormalization-in principle a strongly nonlinear modification of the flow.

\section{Renormalization decreases the energy}

The influence of the renormalization on the exchange energy was for instance investigated in [2] in the continuous case. More precisely, it was shown that for maps $\mathbf{w} \in H^{1}\left(\Omega, \mathbb{R}^{3}\right)$ with $|\mathbf{w}(\mathbf{x})| \geq 1$ a.e. $\mathbf{x} \in \Omega$ one has

$$
\int_{\Omega}\left|\nabla \frac{\mathbf{w}}{|\mathbf{w}|}\right|^{2} \mathrm{~d} \mathbf{x} \leq \int_{\Omega}|\nabla \mathbf{w}|^{2} \mathrm{~d} \mathbf{x} .
$$

Hence, the renormalization step is expected to be energy decreasing — at least as far as the Dirichlet energy is concerned. Applications more related to finite element approximation of micromagnetic configurations can be found in [4]. The discrete version of (12) was proved by Bartels in [7]:

Theorem 1 [7] If the basis functions of the $P^{1}$-approximation satisfy

$$
\forall i \neq j, \int_{\Omega} \nabla \phi_{i}^{h} \cdot \nabla \phi_{j}^{h} \mathrm{~d} \mathbf{x} \leq 0,
$$

then for all $\mathbf{v}=\sum_{i} \mathbf{v}_{i} \phi_{i}^{h} \in V_{h}$ such that $\forall i \in\left\{1, \ldots, N_{h}\right\},\left|\mathbf{v}_{i}\right| \geq 1$ it holds that

$$
\int_{\Omega}\left|\nabla \mathcal{I}_{h}\left(\frac{\mathbf{v}}{|\mathbf{v}|}\right)\right|^{2} \mathrm{~d} \mathbf{x} \leq \int_{\Omega}|\nabla \mathbf{v}|^{2} \mathrm{~d} \mathbf{x} .
$$

In $3 \mathrm{~d}$, the condition (13) — and hence (14) — is for instance satisfied provided all dihedral angles of the tetrahedra of the mesh are smaller than $\pi / 2$, see [17].

\section{The new (almost) order 2-scheme}

Consider an exact solution $\mathbf{m}(n k)$ at time $n k$. As remarked in [1], it is a priori not sufficient to choose $\theta=\frac{1}{2}$ in (11) to achieve quadratic order. Indeed, the renormalization stage inherently introduces an order 2 term due to the expansion 


$$
\mathbf{m}_{n+1}:=\frac{\mathbf{m}(n k)+k \mathbf{v}}{|\mathbf{m}(n k)+k \mathbf{v}|}=\mathbf{m}(n k)+k \mathbf{v}-\frac{k^{2}}{2}|\mathbf{v}|^{2} \mathbf{m}(n k)+O\left(k^{3}\right) .
$$

Hence, in order to have $\mathbf{m}_{n+1}=\mathbf{m}((n+1) k)+O\left(k^{3}\right)$, it is necessary to take this term into account in the variational formulation that defines $\mathbf{v}$. We hence modify the time-discrete approximation of the magnetization $\mathbf{m}$ which we heuristically derive in the following. It is well known that the midpoint rule is exact up to cubic error, i.e.,

$$
\begin{aligned}
\mathbf{m}((n+1) k) & =\mathbf{m}(n k)+k \mathbf{m}_{t}\left(\left(n+\frac{1}{2}\right) k\right)+O\left(k^{3}\right) \\
& =\mathbf{m}(n k)+k\left(\mathbf{m}_{t}(n k)+\frac{k}{2} \mathbf{m}_{t t}(n k)\right)+O\left(k^{3}\right) .
\end{aligned}
$$

Now, setting

$$
\begin{aligned}
\mathbf{v} & =P_{\mathbf{m}^{\perp}}\left(\mathbf{m}_{t}(n k)+\frac{k}{2} \mathbf{m}_{t t}(n k)\right) \\
& =\mathbf{m}_{t}(n k)+\frac{k}{2} P_{\mathbf{m}^{\perp}} \mathbf{m}_{t t}(n k),
\end{aligned}
$$

where $P_{\mathbf{m}^{\perp}}$ denotes the projection onto the orthogonal component of $\mathbf{m}(n k)$, and noticing that $\mathbf{v} \cdot \mathbf{m}(n k)=0$, a Taylor expansion up to cubic order reveals

$$
\begin{aligned}
\frac{\mathbf{m}(n k)+k \mathbf{v}}{|\mathbf{m}(n k)+k \mathbf{v}|}= & \mathbf{m}(n k)+k \mathbf{v}-\frac{k^{2}}{2}|\mathbf{v}|^{2} \mathbf{m}(n k)+O\left(k^{3}\right) \\
= & \mathbf{m}(n k)+k \mathbf{m}_{t}(n k)+\frac{k^{2}}{2} P_{\mathbf{m}^{\perp}}\left(\mathbf{m}_{t t}(n k)\right) \\
& -\frac{k^{2}}{2}\left|\mathbf{m}_{t}(n k)\right|^{2} \mathbf{m}(n k)+O\left(k^{3}\right) \\
= & \mathbf{m}(n k)+k \mathbf{m}_{t}(n k)+\frac{k^{2}}{2} \mathbf{m}_{t t}(n k)+O\left(k^{3}\right) \\
= & \mathbf{m}((n+1) k)+O\left(k^{3}\right) .
\end{aligned}
$$

Notice that we have made use in the above computation that, due to the unit length constraint, one has

$$
\begin{aligned}
\mathbf{m}_{t t}(n k) & =P_{\mathbf{m}^{\perp}}\left(\mathbf{m}_{t t}(n k)\right)+\left(\mathbf{m}_{t t}(n k) \cdot \mathbf{m}(n k)\right) \mathbf{m}(n k) \\
& =P_{\mathbf{m}^{\perp}}\left(\mathbf{m}_{t t}(n k)\right)-\left|\mathbf{m}_{t}(n k)\right|^{2} \mathbf{m}(n k) .
\end{aligned}
$$

We therefore propose to modify the original first order scheme by replacing the tangential update with the higher order approximation (16).

We will use the simplifying shorthand notation $\mathbf{m}=\mathbf{m}(n k)$ and $\mathbf{m}_{t}=\mathbf{m}_{t}(n k)$. Let us proceed with the derivation of the equation that is satisfied by $\mathbf{v}=\mathbf{m}_{t}(n k)+$ $\frac{k}{2} P_{\mathbf{m}^{\perp}} \mathbf{m}_{t t}(n k)$, i.e. the counterpart to (10). The equation will be inferred from the differentiated LLG equation which we restate as 


$$
\alpha \mathbf{m}_{t}+\mathbf{m} \times \mathbf{m}_{t}=\mathbf{H}_{\mathrm{eff}}(\mathbf{m})-\left(\mathbf{H}_{\mathrm{eff}}(\mathbf{m}) \cdot \mathbf{m}\right) \mathbf{m}
$$

by multiplying (1) with $\mathbf{m} \times$.

Remark 1 Although the midpoint rule is of order 2, our scheme is only almost of order 2-as the section's title suggests and as we will see in the sequel. This is due to a regularizing term that is introduced in order to obtain the necessary estimates in the convergence proof. This term prevents the scheme from being of order 2 , in the sense that the consistency error is not of order $O\left(k^{3}\right)$ but only $O\left(k^{3-\epsilon}\right)$ for any $\epsilon>0$. On the other hand, this regularization approach allows for unconditional convergence of the scheme. The approximation in space is still of order 1 . Indeed, only piecewise linear functions ( $P^{1}$ Lagrange finite elements) are used in order to ensure condition (13). It is well known that this condition, which for the time being is mandatory to prove the convergence of the scheme, does not hold for higher order finite elements.

To begin with, the differentiation of (17) w.r.t. time yields

$$
\begin{aligned}
\alpha \mathbf{m}_{t t}+\mathbf{m} \times \mathbf{m}_{t t}= & \frac{\partial \mathbf{H}_{\mathrm{eff}}}{\partial \mathbf{m}}\left(\mathbf{m}_{t}\right)-\left(\frac{\partial \mathbf{H}_{\mathrm{eff}}}{\partial \mathbf{m}}\left(\mathbf{m}_{t}\right) \cdot \mathbf{m}\right) \mathbf{m} \\
& -\left(\mathbf{H}_{\mathrm{eff}}(\mathbf{m}) \cdot \mathbf{m}_{t}\right) \mathbf{m}-\left(\mathbf{H}_{\mathrm{eff}}(\mathbf{m}) \cdot \mathbf{m}\right) \mathbf{m}_{t},
\end{aligned}
$$

where

$$
\frac{\partial \mathbf{H}_{\mathrm{eff}}}{\partial \mathbf{m}}\left(\mathbf{m}_{t}\right)=d^{2} \Delta \mathbf{m}_{t}+\mathbf{H}_{\mathrm{d}}\left(\mathbf{m}_{t}\right)+Q\left(\mathbf{e} \cdot \mathbf{m}_{t}\right) \mathbf{e}
$$

and where we once again used the unit length constraint (5). Now, we compute from (17) and (18).

$$
\begin{aligned}
\alpha \mathbf{v}+\mathbf{m} \times \mathbf{v} & =\alpha \mathbf{m}_{t}+\mathbf{m} \times \mathbf{m}_{t}+\frac{k}{2}\left(\alpha P_{\mathbf{m}^{\perp}} \mathbf{m}_{t t}+\mathbf{m} \times P_{\mathbf{m}^{\perp}} \mathbf{m}_{t t}\right) \\
& =P_{\mathbf{m}^{\perp}}\left(\alpha \mathbf{m}_{t}+\mathbf{m} \times \mathbf{m}_{t}+\frac{k}{2}\left(\alpha \mathbf{m}_{t t}+\mathbf{m} \times \mathbf{m}_{t t}\right)\right) \\
& =P_{\mathbf{m}^{\perp}}\left(\mathbf{H}_{\mathrm{eff}}(\mathbf{m})+\frac{k}{2} \frac{\partial \mathbf{H}_{\mathrm{eff}}}{\partial \mathbf{m}}\left(\mathbf{m}_{t}\right)-\frac{k}{2}\left(\mathbf{H}_{\mathrm{eff}}(\mathbf{m}) \cdot \mathbf{m}\right) \mathbf{m}_{t}\right)
\end{aligned}
$$

that we write in weak form

$$
\begin{aligned}
\int_{\Omega} \alpha \mathbf{v} \cdot \Psi+\mathbf{m} \times \mathbf{v} \cdot \Psi \mathrm{d} \mathbf{x}= & \int_{\Omega} \mathbf{H}_{\mathrm{eff}}(\mathbf{m}) \cdot \Psi \mathrm{d} \mathbf{x}+\frac{k}{2} \int_{\Omega} \frac{\partial \mathbf{H}_{\mathrm{eff}}}{\partial \mathbf{m}}\left(\mathbf{m}_{t}\right) \cdot \Psi \mathrm{d} \mathbf{x} \\
& -\frac{k}{2} \int_{\Omega}\left(\mathbf{H}_{\mathrm{eff}}(\mathbf{m}) \cdot \mathbf{m}\right) \mathbf{m}_{t} \cdot \Psi \mathrm{d} \mathbf{x}
\end{aligned}
$$

for any test function $\Psi$ with $\Psi \cdot \mathbf{m}=0$. Observe that $\mathbf{m}_{t}(n k)=\mathbf{v}+O(k)$, cf. (16). Therefore up to higher order terms 


$$
\begin{aligned}
& \int_{\Omega}\left(\alpha+\frac{k}{2}\left(\mathbf{H}_{\mathrm{eff}}(\mathbf{m}) \cdot \mathbf{m}\right)\right) \mathbf{v} \cdot \Psi+\mathbf{m} \times \mathbf{v} \cdot \Psi \mathrm{d} \mathbf{x}-\frac{k}{2} \int_{\Omega} \frac{\partial \mathbf{H}_{\mathrm{eff}}}{\partial \mathbf{m}}(\mathbf{v}) \cdot \Psi \mathrm{d} \mathbf{x} \\
& \quad=\int_{\Omega} \mathbf{H}_{\mathrm{eff}}(\mathbf{m}) \cdot \Psi \mathrm{d} \mathbf{x}+O\left(k^{2}\right),
\end{aligned}
$$

where we remind that $\mathbf{m}=\mathbf{m}(n k)$ and $\mathbf{m}_{t}=\mathbf{m}_{t}(n k)$. Observe that, if we neglect the residual term, the latter equation is (at first sight surprisingly) linear in v. However, its well-posedness is non-obvious since both the first and the last contributions on the 1.h.s. of (19) potentially affect the definiteness of the symmetric part of the operator. In order to guarantee solvability and uniqueness we proceed with higher order modifications that will finally lead to a well posed formulation. We address the first contribution and define (see Fig. 1)

$$
\tilde{\varphi}_{M}(x)= \begin{cases}\alpha+\frac{k}{2} \min (x, M) & \text { for } x \geq 0, \\ \frac{\alpha}{1+\frac{k}{2 \alpha} \min (-x, M)} & \text { for } x<0 .\end{cases}
$$

Notice that $\tilde{\varphi}_{M}(x)=\alpha+\frac{k}{2} \min (x, M)+O\left(k^{2} M^{2}\right)$. By abuse of notation we define

$$
\varphi_{M}(\mathbf{m})=\tilde{\varphi}_{M}\left(\mathbf{H}_{\mathrm{eff}}(\mathbf{m}) \cdot \mathbf{m}\right)
$$

As long as $\mathbf{H}_{\mathrm{eff}}(\mathbf{m}) \cdot \mathbf{m}$ is uniformly bounded, we derive from (19) by plugging in (21) that

$$
\begin{aligned}
& \int_{\Omega} \varphi_{M}(\mathbf{m}) \mathbf{v} \cdot \Psi+\mathbf{m} \times \mathbf{v} \cdot \Psi \mathrm{d} \mathbf{x}-\frac{k}{2} \int_{\Omega} \frac{\partial \mathbf{H}_{\mathrm{eff}}}{\partial \mathbf{m}}(\mathbf{v}) \cdot \Psi \mathrm{d} \mathbf{x} \\
& \quad=\int_{\Omega} \mathbf{H}_{\mathrm{eff}}(\mathbf{m}) \cdot \Psi \mathrm{d} \mathbf{x}+O\left(k^{2}\right) .
\end{aligned}
$$

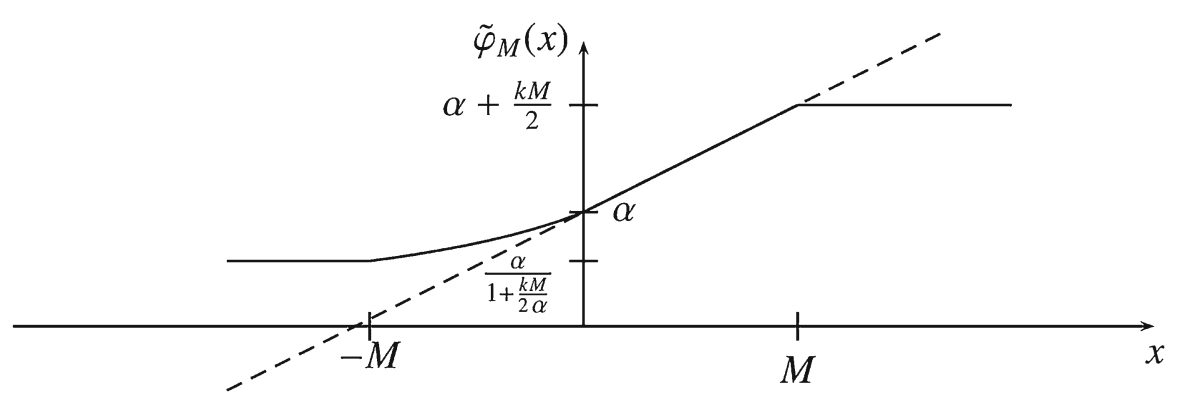

Fig. 1 The regularizing cut-off function $\tilde{\varphi}_{M}(x)$ 
Replacing $\mathbf{H}_{\text {eff }}$ and $\frac{\partial \mathbf{H}_{\text {eff }}}{\partial \mathbf{m}}$ by their very definition, we obtain the counterpart to (10) for our new second order scheme:

$$
\begin{gathered}
\int_{\Omega} \varphi_{M}(\mathbf{m}) \mathbf{v} \cdot \Psi+\mathbf{m} \times \mathbf{v} \cdot \Psi \mathrm{d} \mathbf{x}+\frac{k}{2} \int_{\Omega} d^{2} \nabla \mathbf{v} \cdot \nabla \Psi-\mathbf{H}_{\mathrm{d}}(\mathbf{v}) \cdot \Psi-Q(\mathbf{e} \cdot \mathbf{v})(\mathbf{e} \cdot \Psi) \mathrm{d} \mathbf{x} \\
=\int_{\Omega}-d^{2} \nabla \mathbf{m} \cdot \nabla \Psi+\mathbf{H}_{\mathrm{d}}(\mathbf{m}) \cdot \Psi+Q(\mathbf{e} \cdot \mathbf{m})(\mathbf{e} \cdot \Psi)+\mathbf{H}_{\mathrm{ext}} \cdot \Psi \mathrm{d} \mathbf{x} .
\end{gathered}
$$

We introduce only one further, final modification which implements the strategy delineated in Remark 1: In order to maintain unconditional convergence we additionally modify the second highest order term on the r.h.s. in the following way

$$
\frac{k}{2} \int_{\Omega} d^{2} \nabla \mathbf{v} \cdot \nabla \Psi \mathrm{d} x \rightsquigarrow \frac{k}{2} \int_{\Omega}(1+\rho(k)) d^{2} \nabla \mathbf{v} \cdot \nabla \Psi \mathrm{d} \mathbf{x},
$$

where $\rho(k) \rightarrow 0$ as $k \rightarrow 0$. Take note that for $\rho$ decreasing at least linearly, quadratic order is conserved. However, only in case that $\rho$ is slightly sublinear, for example $\rho(k)=k|\ln (k)|$, do we in fact achieve unconditional convergence.

Adopting Algorithm 1, we arrive at the following scheme:

Algorithm 2 Given an initial $\mathbf{m}^{0} \in M_{h}$ choose a time step size $k=\frac{T}{N}$ with $N \in \mathbb{N}$ and appropriate $\rho(k)$ and $M$, cf. Theorem 2 . For $n=0,1, \ldots, N$

$$
\left[\begin{array}{l}
\text { a) Find } \mathbf{v}^{n} \in K_{\mathbf{m}^{n}} \text { such that for all test functions } \Psi \in K_{\mathbf{m}^{n}} \\
\quad+\frac{k}{2} \int_{\Omega}(1+\rho(k)) d^{2} \nabla \mathbf{v}^{n} \cdot \nabla \Psi-\mathbf{H}_{\mathrm{d}}\left(\mathbf{v}^{n}\right) \cdot \Psi-Q\left(\mathbf{e} \cdot \mathbf{v}^{n}\right)(\mathbf{e} \cdot \Psi) \mathrm{d} \mathbf{x} \\
=\int_{\Omega}-d^{2} \nabla \mathbf{m}^{n} \times \mathbf{v}^{n} \cdot \Psi \mathrm{d} \mathbf{x} \\
\text { b) Set } \mathbf{m}^{n+1}=\sum_{i} \mathbf{m}_{i}^{n+1} \phi_{i}^{h}, \text { where } \forall i, \mathbf{m}_{i}^{n+1}=\frac{\left.\left.\mathbf{m}_{i}^{n}+k \mathbf{m}_{i}^{n}\right)+\mathbf{H}_{\mathrm{ext}}+\mathbf{H}_{\mathrm{aniso}}\left(\mathbf{m}^{n}\right)\right) \cdot \Psi \mathrm{d} \mathbf{x} .}{\left|\mathbf{m}_{i}^{n}+k \mathbf{v}_{i}^{n}\right|}
\end{array}\right.
$$

The appropriate choice of $\rho$ and $M$ can be inferred from our convergence result, see Theorem 2.

Let us sum up: the new scheme replaces the search of $\mathbf{v}$ as solution to (11) by the search of $\mathbf{v}$ as a solution to (24). Besides this substitution, the algorithm outlined in Sect. 4 remains as before in the sense that the renormalization and the interpolation w.r.t. time are left unchanged. Since Eq. (24) is linear in $\mathbf{v}$, our algorithm is very favorable in practice. 
Before we state our theorem about the convergence let us explicitly make a statement about its order.

Proposition 1 Consider a smooth (in space and time) solution $\mathbf{m}$ to (1) at time $t=n k$ and a semi-discrete time-approximation to $m$ at time $t+k$ on the basis of (24). More precisely, given $\mathbf{m}$ at time $t=n k$ determine $\mathbf{v}$ as a solution to the variational formulation (24) with $\rho(k)=0$ and $M(k)$ sufficiently large and set

$$
\tilde{\mathbf{m}}(\mathbf{x}, t+k)=\frac{\mathbf{m}(\mathbf{x}, t)+k \mathbf{v}(\mathbf{x}, t)}{|\mathbf{m}(\mathbf{x}, t)+k \mathbf{v}(\mathbf{x}, t)|} \quad \text { for all } \mathbf{x} \in \Omega
$$

Then $\tilde{\mathbf{m}}(t+k)$ approximates $\mathbf{m}(t+k)$ up to cubic error in $k$.

Argument for Proposition 1 The proof is a direct consequence of the Taylor expansion performed in (16).

Remark 2 The smoothness of solutions to (1) has been widely studied during the past years. In general, the formation of singularities cannot be ruled out and we can usually not assume that a solution to the initial value problem will be regular. Our statement about the order of the approximation is thus only a first little step on the way to a proof of the order of convergence, which is way beyond the scope of this paper. Let us insist on the fact that, to the knowledge of the authors, there does not yet exist any numerical scheme for LLG equations for which an order of convergence can be proven.

Let us now turn to the convergence result.

Theorem 2 Let $\mathbf{m}_{0} \in H^{1}\left(\Omega, S^{2}\right)$. Suppose $\mathbf{m}^{0} \rightarrow \mathbf{m}_{0}$ in $H^{1}(\Omega)$ as $h \rightarrow 0$. If the regular sequence of conformal triangulations $\left(\mathcal{T}_{h}\right)_{h>0}$ satisfies condition (13), then the approximation $\left(\mathbf{m}_{h, k}\right)$ of the sequence constructed via Algorithm 2 and interpolated according to Definition 1 converges (up to the extraction of a subsequence) weakly in $H^{1}\left(\Omega_{T}\right)$ to a weak solution $\mathbf{m}$ of (1) as $h$ and $k$ tend to 0 provided $\rho(k) \rightarrow_{k \rightarrow 0} 0$ and one of the two following conditions hold:

$-k^{-1} \rho(k) \rightarrow{ }_{(h, k) \rightarrow 0} \infty$ and $k M \rightarrow{ }_{(h, k) \rightarrow 0} 0$ or

$-\rho \equiv 0$ and $k h^{-1} \rightarrow 0$ as $(h, k) \rightarrow 0$.

Proof of Theorem 2 As stated before, the proof consists of two main steps: establishing estimates which guarantee the existence of a sufficiently strong converging subsequence, then proving that the latter converges indeed to a solution (which satisfies the energy estimate). Several arguments in the proof have already been used in e.g. $[1,3,8]$, but we restate them for the self-consistency of the paper. We will need the following classical estimate from elliptic regularity theory, namely

$$
\left\|\mathbf{H}_{\mathrm{d}}(\mathbf{m})\right\|_{L^{p}(\Omega)} \leq C\|\mathbf{m}\|_{L^{p}(\Omega)},
$$

for all $p \in(1,+\infty)$ and a positive constant $C$ which depends only on $p$. 
Bounds on the sequence As we have already observed, the variational formulation in the iteration of (23) has a unique solution $\mathbf{v}^{n}$. We test the equation with $\Psi=\mathbf{v}^{n}$ itself to find that

$$
\begin{aligned}
& \int_{\Omega} \varphi_{M}\left(\mathbf{m}^{n}\right)\left|\mathbf{v}^{n}\right|^{2} \mathrm{~d} \mathbf{x}+\frac{k}{2} \int_{\Omega}(1+\rho(k)) d^{2}\left|\nabla \mathbf{v}^{n}\right|^{2}-\mathbf{H}_{\mathrm{d}}\left(\mathbf{v}^{n}\right) \cdot \mathbf{v}^{n}-Q\left(\mathbf{v}^{n} \cdot e\right)^{2} \mathrm{~d} \mathbf{x} \\
& =\int_{\Omega}-d^{2} \nabla \mathbf{m}^{n} \cdot \nabla \mathbf{v}^{n}+\mathbf{H}_{\mathrm{d}}\left(\mathbf{m}^{n}\right) \cdot \mathbf{v}^{n}+Q\left(\mathbf{e} \cdot \mathbf{m}^{n}\right)\left(\mathbf{e} \cdot \mathbf{v}^{n}\right)+\mathbf{H}_{\mathrm{ext}} \cdot \mathbf{v}^{n} \mathrm{~d} \mathbf{x} .
\end{aligned}
$$

Since we assume that the triangulation $\mathcal{T}_{h}$ satisfies the angle condition (13) we have that

$$
\begin{aligned}
\int_{\Omega}\left|\nabla \mathbf{m}^{n+1}\right|^{2} \mathrm{~d} \mathbf{x} & \leq \int_{\Omega}\left|\nabla\left(\mathbf{m}^{n}+k \mathbf{v}^{n}\right)\right|^{2} \mathrm{~d} \mathbf{x} \\
& \leq \int_{\Omega}\left|\nabla \mathbf{m}^{n}\right|^{2} \mathrm{~d} \mathbf{x}+2 k \int_{\Omega} \nabla \mathbf{m}^{n} \cdot \nabla \mathbf{v}^{n} \mathrm{~d} \mathbf{x}+k^{2} \int_{\Omega}\left|\nabla \mathbf{v}^{n}\right|^{2} \mathrm{~d} \mathbf{x} .
\end{aligned}
$$

Using (26) we obtain that

$$
\begin{aligned}
d^{2} \int_{\Omega}\left|\nabla \mathbf{m}^{n+1}\right|^{2} \mathrm{~d} \mathbf{x} \leq & d^{2} \int_{\Omega}\left|\nabla \mathbf{m}^{n}\right|^{2} \mathrm{~d} \mathbf{x}-2 k \int_{\Omega} \varphi_{M}\left(\mathbf{m}^{n}\right)\left|\mathbf{v}^{n}\right|^{2} \mathrm{~d} \mathbf{x} \\
& +k^{2} \int_{\Omega}\left(\mathbf{H}_{\mathrm{d}}\left(\mathbf{v}^{n}\right) \cdot \mathbf{v}^{n}+Q\left(\mathbf{e} \cdot \mathbf{v}_{n}\right)^{2}\right) \mathrm{d} \mathbf{x} \\
& +2 k \int_{\Omega}\left(\mathbf{H}_{\mathrm{d}}\left(\mathbf{m}^{n}\right)+\mathbf{H}_{\text {aniso }}\left(\mathbf{v}^{n}\right)+\mathbf{H}_{\mathrm{ext}}\right) \cdot \mathbf{v}^{n} \mathrm{~d} \mathbf{x} \\
& -k^{2} \rho(k) d^{2} \int_{\Omega}\left|\nabla \mathbf{v}^{n}\right|^{2} \mathrm{~d} \mathbf{x} .
\end{aligned}
$$

Before we move on, let us just rewrite the latter estimate as

$$
\begin{aligned}
d^{2} \int_{\Omega}\left|\nabla \mathbf{m}^{n+1}\right|^{2} \mathrm{~d} \mathbf{x} \leq & d^{2} \int_{\Omega}\left|\nabla \mathbf{m}^{n}\right|^{2} \mathrm{~d} \mathbf{x}-2 k \int_{\Omega} \varphi_{M}\left(\mathbf{m}^{n}\right)\left|\mathbf{v}^{n}\right|^{2} \mathrm{~d} \mathbf{x} \\
& +k^{2} \int_{\Omega} \frac{\partial \overline{\mathbf{H}}_{\mathrm{eff}}}{\partial \mathbf{m}}\left(\mathbf{v}^{n}\right) \cdot \mathbf{v}^{n} \mathrm{~d} \mathbf{x}+2 k \int_{\Omega} \overline{\mathbf{H}}_{\mathrm{eff}}\left(\mathbf{m}^{n}\right) \cdot \mathbf{v}^{n} \mathrm{~d} \mathbf{x} \\
& -k^{2} \rho(k) d^{2} \int_{\Omega}\left|\nabla \mathbf{v}^{n}\right|^{2} \mathrm{~d} \mathbf{x} .
\end{aligned}
$$

We partially neglect the negative contributions on the r.h.s. of (28)—-those which are quadratic in $\mathbf{v}^{n}$ - and use (25) to obtain 


$$
\begin{aligned}
d^{2} \int_{\Omega}\left|\nabla \mathbf{m}^{n+1}\right|^{2} \mathrm{~d} \mathbf{x} \leq & d^{2} \int_{\Omega}\left|\nabla \mathbf{m}^{n}\right|^{2} \mathrm{~d} \mathbf{x}-2 k \int_{\Omega} \varphi_{M}(\mathbf{m})\left|\mathbf{v}^{n}\right|^{2} \mathrm{~d} \mathbf{x} \\
& +2 k|| \overline{\mathbf{H}}_{\mathrm{eff}}\left(\mathbf{m}^{n}\right)||_{L^{2}}|| \mathbf{v}^{n} \|_{L^{2}}-k^{2} \rho(k) d^{2} \int_{\Omega}\left|\nabla \mathbf{v}^{n}\right|^{2} \mathrm{~d} \mathbf{x} \\
\leq & d^{2} \int_{\Omega}\left|\nabla \mathbf{m}^{n}\right|^{2} \mathrm{~d} \mathbf{x}-2 k \int_{\Omega} \varphi_{M}(\mathbf{m})\left|\mathbf{v}^{n}\right|^{2} \mathrm{~d} \mathbf{x} \\
& +C k\left\|\mathbf{v}_{n}\right\|_{L^{2}}-k^{2} \rho(k) d^{2} \int_{\Omega}\left|\nabla \mathbf{v}^{n}\right|^{2} \mathrm{~d} \mathbf{x}
\end{aligned}
$$

where the generic constant $C$ depends on $Q$ and $|\Omega|$. Due to Young's inequality, we have that $C k\left\|\mathbf{v}_{n}\right\|_{L^{2}(\Omega)} \leq k \beta\left\|\mathbf{v}_{n}\right\|_{L^{2}(\Omega)}^{2}+\frac{k C^{2}}{4 \beta}$ for $\beta>0$. Using the uniform bound

$$
\varphi_{M}(\mathbf{m}) \geq \beta=\frac{\alpha}{1+\frac{k}{2} M}
$$

we find by rewriting (29) that

$$
\begin{aligned}
& d^{2} \int_{\Omega}\left|\nabla \mathbf{m}^{n+1}\right|^{2} \mathrm{~d} \mathbf{x}+\left.\beta k|| \mathbf{v}^{n}\right|_{L^{2}(\Omega)} ^{2}+k^{2} \rho(k) d^{2} \int_{\Omega}\left|\nabla \mathbf{v}^{n}\right|^{2} \mathrm{~d} \mathbf{x} \\
& \leq d^{2} \int_{\Omega}\left|\nabla \mathbf{m}^{n}\right|^{2} \mathrm{~d} \mathbf{x}+\frac{k C^{2}(Q,|\Omega|)}{4 \beta} .
\end{aligned}
$$

Summing up in (30) over the time steps we find that

$$
\begin{aligned}
& d^{2} \int_{\Omega}\left|\nabla \mathbf{m}^{N}\right|^{2} \mathrm{~d} \mathbf{x}+\beta k \sum_{n=0}^{N-1} \int_{\Omega}\left|\mathbf{v}^{n}\right|^{2} \mathrm{~d} \mathbf{x}+k^{2} \rho(k) d^{2} \int_{\Omega}\left|\nabla \mathbf{v}^{n}\right|^{2} \mathrm{~d} \mathbf{x} \\
& \leq C\left(T, d^{2} \int_{\Omega}\left|\nabla \mathbf{m}_{0}\right|^{2} \mathrm{~d} \mathbf{x}, \beta, Q, \mathbf{H}_{\text {aniso }}\right)
\end{aligned}
$$

From now on, most of the arguments follow the same line as in [1]. It holds that

$$
\left|\frac{\mathbf{m}_{i}^{n+1}-\mathbf{m}_{i}^{n}}{k}\right| \leq\left|\mathbf{v}_{i}^{n}\right| \text {, for all } n \leq N, \quad \text { and } \quad i \in\left\{1, \cdots, N_{h}\right\} \text {. }
$$

Moreover, there exists $c>0$ such that for all $1 \leq p<+\infty$ and all $\phi_{h} \in V_{h}$ there holds

$$
\frac{1}{c}\left\|\phi_{h}\right\|_{L^{p}(\Omega)}^{p} \leq h^{d} \sum_{i}\left|\phi_{h}\left(x_{i}^{h}\right)\right|^{p} \leq c\left\|\phi_{h}\right\|_{L^{p}(\Omega)}^{p}
$$


which implies

$$
\left\|\frac{\mathbf{m}^{n+1}-\mathbf{m}^{n}}{k}\right\|_{L^{2}} \leq c^{2}\left\|\mathbf{v}^{n}\right\|_{L^{2}} .
$$

Hence we obtain from the energy estimate (31) using (33) the following bounds

$$
\begin{gathered}
\mathbf{m}_{h, k} \text { is uniformly bounded in } H^{1}\left(\Omega_{T}\right), \\
\mathbf{v}_{h, k} \text { is uniformly bounded in } L^{2}\left(\Omega_{T}\right) .
\end{gathered}
$$

Due to (34) and (35), there exist $\overline{\mathbf{m}} \in H^{1}\left(\Omega_{T}\right)$ and $\mathbf{v} \in L^{2}\left(\Omega_{T}\right)$ such that up to the extraction of subsequences

$$
\begin{aligned}
& \mathbf{m}_{h, k} \rightarrow_{(h, k) \rightarrow 0} \overline{\mathbf{m}} \text { weakly in } H^{1}\left(\Omega_{T}\right), \\
& \mathbf{m}_{h, k} \rightarrow_{(h, k) \rightarrow 0} \overline{\mathbf{m}} \text { strongly in } L^{2}\left(\Omega_{T}\right), \\
& \mathbf{v}_{h, k} \rightarrow_{(h, k) \rightarrow 0} \mathbf{v} \text { weakly in } L^{2}\left(\Omega_{T}\right) .
\end{aligned}
$$

In addition, we have from (31) that

$$
\sum_{n=0}^{N-1} k^{2} \rho(k) \int_{\Omega}\left|\nabla \mathbf{v}^{n}\right|^{2} \mathrm{~d} \mathbf{x}=k \rho(k) \int_{0}^{T} \int_{\Omega}\left|\nabla \mathbf{v}_{h, k}\right|^{2} \mathrm{~d} \mathbf{x} \leq C<+\infty .
$$

If $\rho$ decreases only sublinearly, i.e. $k^{-1} \rho(k) \rightarrow_{k \rightarrow 0}+\infty$, we deduce that

$$
k\|\nabla \mathbf{v}\|_{L^{2}\left(\Omega_{T}\right)} \rightarrow{ }_{(h, k) \rightarrow 0} 0 .
$$

If $\rho$ decreases linearly or faster we have to resort to the inverse estimate $\|\nabla \mathbf{v}\|_{L^{2}\left(\Omega_{T}\right)} \lesssim$ $\frac{1}{h}\|\mathbf{v}\|_{L^{2}\left(\Omega_{T}\right)}$ so that (39) holds true if $k h^{-1} \rightarrow 0$ when both $(h, k) \rightarrow 0$.

Preliminary estimates We want to prove that $\overline{\mathbf{m}}$ satisfies (7) and follow the strategy of [1]. To begin with, we restate some further estimates from [1] and derive some necessary statements about convergence. Observe that for all $n=0, \cdots, J$ and all $t \in[n k,(n+1) k)$

$$
\left|\mathbf{m}_{h, k}(\mathbf{x}, t)-\mathbf{m}_{h, k}^{-}(\mathbf{x}, t)\right|=\left|(t-n k)\left(\frac{\mathbf{m}^{n+1}(\mathbf{x})-\mathbf{m}^{n}(\mathbf{x})}{k}\right)\right| \leq k\left|\partial_{t} \mathbf{m}_{h, k}(\mathbf{x}, t)\right| .
$$

Therefore

$$
\left\|\mathbf{m}_{h, k}-\mathbf{m}_{h, k}^{-}\right\|_{L^{2}\left(\Omega_{T}\right)} \leq k\left\|\partial_{t} \mathbf{m}_{h, k}\right\|_{L^{2}\left(\Omega_{T}\right)} \rightarrow_{(h, k) \rightarrow 0} 0,
$$

which entails that

$$
\mathbf{m}_{h, k}^{-} \rightarrow(h, k) \rightarrow 0 \overline{\mathbf{m}} \text { strongly in } L^{2}\left(\Omega_{T}\right)
$$


Moreover, on any tetrahedron $K$ of $\mathcal{T}_{h}$, and for any $\mathbf{u} \in M_{h}$, and any vertex $\mathbf{x}_{i}^{h}$ of $K$, one has

$$
|| \mathbf{u}(\mathbf{x})|-| \mathbf{u}\left(\mathbf{x}_{i}^{h}\right)||^{2} \leq C h^{2}|\nabla \mathbf{u}|^{2}
$$

(recall that $\nabla \mathbf{u}$ is constant on $K$ ), from which one deduces (since $\left|\mathbf{m}_{h, k}^{-}\left(\mathbf{x}_{i}^{h}\right)\right|=1$ )

$$
\int_{\Omega_{T}}|1-| \mathbf{m}_{h, k}^{-}||^{2} \mathrm{~d} \mathbf{x} \leq C h^{2}\left\|\nabla \mathbf{m}_{h, k}^{-}\right\|_{L^{2}\left(\Omega_{T}\right)}^{2} .
$$

This shows that $|\overline{\mathbf{m}}(\mathbf{x}, t)|=1$ a.e. $(\mathbf{x}, t) \in \Omega_{T}$.

Eventually, from the fact that at each vertex $\forall i \in\left\{1, \ldots, N_{h}\right\}$

$$
\left|\mathbf{m}_{i}^{n+1}-\mathbf{m}_{i}^{n}-k \mathbf{v}_{i}^{n}\right|=\left|\mathbf{m}_{i}^{n}+k \mathbf{v}_{i}^{n}\right|-1 \leq \frac{1}{2} k^{2}\left|\mathbf{v}_{i}^{n}\right|^{2},
$$

we derive

$$
\left|\frac{\mathbf{m}_{i}^{n+1}-\mathbf{m}_{i}^{n}}{k}-\mathbf{v}_{i}^{n}\right| \leq \frac{1}{2} k\left|\mathbf{v}_{i}^{n}\right|^{2} .
$$

Appealing to (32) the latter entails that

$$
\left\|\partial_{t} \mathbf{m}_{h, k}-\mathbf{v}_{h, k}\right\|_{L^{1}\left(\Omega_{T}\right)} \leq c^{2} k\left\|\mathbf{v}_{h, k}\right\|_{L^{2}\left(\Omega_{T}\right)}^{2} \rightarrow_{(h, k) \rightarrow 0} 0 .
$$

This is sufficient to conclude that $\mathbf{v}=\partial_{t} \overline{\mathbf{m}}$ in (38).

General properties of interpolation operator Before we start with the penultimate step of proving convergence, let us state some general properties of the nodal interpolation operator which we repeatedly use in the sequel. Up to dimension three, there holds for any function $\varphi \in H^{2}(\Omega) \subset \mathcal{C}^{0}(\bar{\Omega})$

$$
\left\|\varphi-\mathcal{I}_{h}(\varphi)\right\|_{H^{1}(\Omega)} \leq C h\left\|\nabla^{2} \varphi\right\|_{L^{2} \Omega}
$$

Since the basis functions are linear on each triangle one can deduce from (41) that

$$
\left\|\mathbf{m}_{h, k}^{-} \times \tilde{\Psi}-\mathcal{I}_{h}\left(\mathbf{m}_{h, k}^{-} \times \tilde{\Psi}\right)\right\|_{L^{2}\left([0, T], H^{1}\right)} \leq C h\left\|\mathbf{m}_{h, k}^{-}\right\|_{H^{1}\left(\Omega_{T}\right)}\|\Psi\|_{W^{2, \infty}}
$$

see [1, p.7].

Convergence to a solution of the LLG equation Having established the preliminary results above, we are now ready to proceed with the proof of convergence. We recall 
that $\mathcal{I}_{h}$ is the nodal interpolation, cf. (9), and test (24) with $\Psi=\mathcal{I}_{h}\left(\mathbf{m}_{h, k}^{-} \times \tilde{\Psi}\right)$ where $\tilde{\Psi} \in C_{0}^{\infty}\left(\Omega_{T}\right)^{3}$. After suitable integration in time we get

$$
\begin{aligned}
& \int_{\Omega_{T}} \varphi_{M}\left(\mathbf{m}_{h, k}^{-}\right) \mathbf{v}_{h, k} \cdot \mathcal{I}_{h}\left(\mathbf{m}_{h, k}^{-} \times \tilde{\Psi}\right) \mathrm{d} \mathbf{x} \mathrm{d} t+\int_{\Omega_{T}} \mathbf{m}_{h, k}^{-} \times \mathbf{v}_{h, k} \cdot \mathcal{I}_{h}\left(\mathbf{m}_{h, k}^{-} \times \tilde{\Psi}\right) \mathrm{d} \mathbf{x} \mathrm{d} t \\
& \quad+\frac{k}{2} \int_{\Omega_{T}}(1+\rho(k)) d^{2} \nabla \mathbf{v}_{h, k} \cdot \nabla \mathcal{I}_{h}\left(\mathbf{m}_{h, k}^{-} \times \tilde{\Psi}\right)-\mathbf{H}_{\mathrm{d}}\left(\mathbf{v}_{h, k}\right) \cdot \mathcal{I}_{h}\left(\mathbf{m}_{h, k}^{-} \times \tilde{\Psi}\right) \\
& \quad-Q\left(\mathbf{e} \cdot \mathbf{v}_{h, k}\right)\left(\mathbf{e} \cdot \mathcal{I}_{h}\left(\mathbf{m}_{h, k}^{-} \times \tilde{\Psi}\right)\right) \mathrm{d} \mathbf{x} \mathrm{d} t \\
& =\int_{\Omega_{T}}-d^{2} \nabla \mathbf{m}_{h, k}^{-} \cdot \nabla \mathcal{I}_{h}\left(\mathbf{m}_{h, k}^{-} \times \tilde{\Psi}\right)+\mathbf{H}_{\mathrm{d}}\left(\mathbf{m}_{h, k}\right) \cdot \mathcal{I}_{h}\left(\mathbf{m}_{h, k}^{-} \times \tilde{\Psi}\right) \\
& \quad+Q\left(\mathbf{e} \cdot \mathbf{m}_{h, k}\right)\left(\mathbf{e} \cdot \mathcal{I}_{h}\left(\mathbf{m}_{h, k}^{-} \times \tilde{\Psi}\right)\right)+H_{\mathrm{ext}} \cdot \mathcal{I}_{h}\left(\mathbf{m}_{h, k}^{-} \times \tilde{\Psi}\right) \mathrm{d} \mathbf{x} \mathrm{d} t .
\end{aligned}
$$

Our goal is to pass to the limit $(k, h) \rightarrow 0$ in the latter equation (43) to recover the LLG equation (10). As we shall see, the first and the third term on the 1.h.s. and the first term on the r.h.s. are a little bit subtle and have to be treated with caution. The remaining contributions behave well under the established convergence; this is particularly due to the fact that $\mathbf{H}_{\mathrm{d}}$ is $L^{2}$-continuous. For the second contribution on the 1.h.s. one further uses that the $L^{\infty}$ bound on $\mathbf{m}^{-}$improves (37) to strong convergence in any $L^{p}$ with $1<p<+\infty$.

Observing that $\left|\varphi_{M}\right|$ is uniformly bounded, and that $\left|\varphi_{M}-\alpha\right| \leq \frac{k M}{2}$, as long as $k M \rightarrow 0$ for both $(h, k) \rightarrow 0$ the strong convergence of $\mathbf{m}_{h, k}^{-}$is sufficient to conclude that

$$
\int_{\Omega_{T}} \varphi_{M}\left(\mathbf{m}_{h, k}^{-}\right) \mathbf{v}_{h, k} \cdot \mathcal{I}_{h}\left(\mathbf{m}_{h, k}^{-} \times \tilde{\Psi}\right) \mathrm{d} \mathbf{x} \mathrm{d} t \rightarrow_{(h, k) \rightarrow 0} \alpha \int_{\Omega_{T}} \mathbf{v} \cdot(\overline{\mathbf{m}} \times \tilde{\Psi}) \mathrm{d} \mathbf{x} \mathrm{d} t .
$$

Indeed, using the triangle inequality we find that

$$
\begin{aligned}
& \left|\int_{\Omega_{T}} \varphi_{M}\left(\mathbf{m}_{h, k}^{-}\right) \mathbf{v}_{h, k}^{n} \cdot \mathcal{I}_{h}\left(\mathbf{m}_{h, k}^{-} \times \tilde{\Psi}\right) \mathrm{d} \mathbf{x} \mathrm{d} t-\alpha \int_{\Omega_{T}} \mathbf{v} \cdot(\overline{\mathbf{m}} \times \tilde{\Psi}) \mathrm{d} \mathbf{x} \mathrm{d} t\right| \\
& \leq\left|\int_{\Omega_{T}} \varphi_{M}\left(\mathbf{m}_{h, k}^{-}\right) \mathbf{v}_{h, k} \cdot\left(\mathbf{m}_{h, k}^{-} \times \tilde{\Psi}\right) \mathrm{d} \mathbf{x} \mathrm{d} t-\alpha \int_{\Omega_{T}} \mathbf{v} \cdot(\overline{\mathbf{m}} \times \tilde{\Psi}) \mathrm{d} \mathbf{x} \mathrm{d} t\right| \\
& \quad+\left|\int_{\Omega_{T}} \varphi_{M}\left(\mathbf{m}_{h, k}^{-}\right) \mathbf{v}_{h, k} \cdot\left(\left(\mathbf{m}_{h, k}^{-} \times \tilde{\Psi}\right)-\mathcal{I}_{h}\left(\mathbf{m}_{h, k}^{-} \times \tilde{\Psi}\right)\right) \mathrm{d} \mathbf{x} \mathrm{d} t\right|
\end{aligned}
$$


The first term tends to zero since

$$
\begin{array}{ll}
\varphi_{M}\left(\mathbf{m}^{-}\right) \rightarrow_{(h, k) \rightarrow 0} \alpha & \text { in } L^{\infty}(\Omega), \\
\mathbf{m}_{h, k}^{-} \rightarrow_{(h, k) \rightarrow 0} \overline{\mathbf{m}} \text { in } & L^{2}\left(\Omega_{T}\right), \text { and } \\
\mathbf{v}_{h, k} \rightarrow_{(h, k) \rightarrow 0} \mathbf{v}=\frac{\partial \overline{\mathbf{m}}}{\partial t} & \text { in } L^{2}\left(\Omega_{T}\right)
\end{array}
$$

as $h, k \rightarrow 0$.

Since $\varphi_{M}\left(\mathbf{m}_{h, k}^{-}\right)$is uniformly bounded, we can evoke (42) to obtain that the second contribution tends to zero. This establishes (44).

Let's turn to the next term in (43). Convergence here essentially relies upon the estimate (39). In fact, appealing once again to (42) we see that instead of establishing

$$
\frac{k}{2} d^{2} \int_{\Omega_{T}}(1+\rho(k)) \nabla \mathbf{v}_{h, k} \cdot \nabla \mathcal{I}_{h}\left(\mathbf{m}_{h, k}^{-} \times \tilde{\Psi}\right) \mathrm{d} \mathbf{x} \rightarrow_{(h, k) \rightarrow 0} 0,
$$

it suffices to show

$$
\frac{k}{2} d^{2} \int_{\Omega_{T}}(1+\rho(k)) \nabla \mathbf{v}_{h, k} \cdot \nabla\left(\mathbf{m}_{h, k}^{-} \times \tilde{\Psi}\right) \mathrm{d} \mathbf{x} \rightarrow_{(h, k) \rightarrow 0} 0,
$$

which obviously follows from (39) by Young's inequality.

Finally, the convergence of the last term in (45) follows from the orthogonality property of the cross product and (36), (37) by once again appealing to (42) since

$$
\begin{aligned}
& \frac{k}{2}\left|\int_{\Omega_{T}} \nabla \mathbf{m}_{h, k}^{-} \cdot \nabla \mathcal{I}_{h}\left(\mathbf{m}_{h, k}^{-} \times \tilde{\Psi}\right) \mathrm{d} \mathbf{x} \mathrm{d} t-\int_{\Omega_{T}} \nabla \overline{\mathbf{m}} \cdot \overline{\mathbf{m}} \times \nabla \tilde{\Psi} \mathrm{d} \mathbf{x} \mathrm{d} t\right| \\
& \quad \leq \frac{k}{2}\left|\int_{\Omega_{T}} \nabla \mathbf{m}_{h, k}^{-} \cdot \nabla\left(\mathcal{I}_{h}\left(\mathbf{m}_{h, k}^{-} \times \tilde{\Psi}\right)-\left(\mathbf{m}_{h, k}^{-} \times \tilde{\Psi}\right)\right) \mathrm{d} \mathbf{x} \mathrm{d} t\right| \\
& +\frac{k}{2}\left|\int_{\Omega_{T}} \nabla \mathbf{m}_{h, k}^{-} \cdot \nabla\left(\mathbf{m}_{h, k}^{-} \times \tilde{\Psi}\right)-\nabla \overline{\mathbf{m}} \cdot \overline{\mathbf{m}} \times \nabla \tilde{\Psi} \mathrm{d} \mathbf{x} \mathrm{d} t\right| \\
& \quad=\frac{k}{2}\left|\int_{\Omega_{T}} \nabla \mathbf{m}_{h, k}^{-} \cdot \nabla\left(\mathcal{I}_{h}\left(\mathbf{m}_{h, k}^{-} \times \tilde{\Psi}\right)-\left(\mathbf{m}_{h, k}^{-} \times \tilde{\Psi}\right)\right) \mathrm{d} \mathbf{x} \mathrm{d} t\right| \\
& +\frac{k}{2}\left|\int_{\Omega_{T}} \nabla \mathbf{m}_{h, k}^{-} \cdot\left(\mathbf{m}_{h, k}^{-} \times \nabla \tilde{\Psi}\right)-\nabla \overline{\mathbf{m}} \cdot(\overline{\mathbf{m}} \times \nabla \tilde{\Psi}) \mathrm{d} \mathbf{x} \mathrm{d} t\right|
\end{aligned}
$$


Energy estimate We finally establish the energy estimate. From (27) we deduce that $\forall n \in\left\{0, \cdots, N_{h}\right\}$

$$
\begin{aligned}
\mathcal{E}\left(\mathbf{m}^{n+1}\right)-\mathcal{E}\left(\mathbf{m}^{n}\right) \leq & -k \int_{\Omega} \varphi\left(\mathbf{m}^{n}\right)\left|\mathbf{v}^{n}\right|^{2} \mathrm{~d} \mathbf{x}+k \int_{\Omega} \overline{\mathbf{H}}_{\mathrm{eff}}\left(\mathbf{m}^{n}\right) \cdot \mathbf{v}^{n} \mathrm{~d} \mathbf{x} \\
& +\frac{k^{2}}{2} \int_{\Omega} \frac{\partial \overline{\mathbf{H}}_{\mathrm{eff}}}{\partial \mathbf{m}}\left(\mathbf{v}^{n}\right) \cdot \mathbf{v}^{n} \mathrm{~d} \mathbf{x}-\frac{k^{2}}{2} \rho(k) d^{2} \int_{\Omega}\left|\nabla \mathbf{v}^{n}\right|^{2} \mathrm{~d} \mathbf{x} \\
& -\frac{1}{2} \int_{\Omega} \overline{\mathbf{H}}_{\mathrm{eff}}\left(\mathbf{m}^{n+1}+\mathbf{m}^{n}\right) \cdot\left(\mathbf{m}^{n+1}-\mathbf{m}^{n}\right) \mathrm{d} \mathbf{x}
\end{aligned}
$$

cf. (3). Let us introduce the shorthand $\overline{\mathbf{H}}_{\text {eff }}^{n}=\overline{\mathbf{H}}_{\text {eff }}\left(\mathbf{m}^{n}\right)$ for the remaining effective field. We consider the contributions in (49) separately and start with the observation that

$$
\begin{aligned}
& k \int_{\Omega} \overline{\mathbf{H}}_{\mathrm{eff}}^{n} \cdot \mathbf{v}^{n} \mathrm{~d} \mathbf{x}-\frac{1}{2} \int_{\Omega}\left(\overline{\mathbf{H}}_{\mathrm{eff}}^{n+1}+\overline{\mathbf{H}}_{\mathrm{eff}}^{n}\right) \cdot\left(\mathbf{m}^{n+1}-\mathbf{m}^{n}\right) \mathrm{d} \mathbf{x} \\
& =\int_{\Omega} \overline{\mathbf{H}}_{\mathrm{eff}}^{n} \cdot\left(\mathbf{m}^{n+1}-\mathbf{m}^{n}-k \mathbf{v}^{n}\right) \mathrm{d} \mathbf{x}+\frac{1}{2} \int_{\Omega}\left(\overline{\mathbf{H}}_{\mathrm{eff}}^{n+1}-\overline{\mathbf{H}}_{\mathrm{eff}}^{n}\right) \cdot\left(\mathbf{m}^{n+1}-\mathbf{m}^{n}\right) \mathrm{d} \mathbf{x} .
\end{aligned}
$$

Hence due to (33) and (40) combined with (32)

$$
\begin{aligned}
& \left|k \int_{\Omega} \overline{\mathbf{H}}_{\mathrm{eff}}^{n} \cdot \mathbf{v}^{n} \mathrm{~d} \mathbf{x}-\frac{1}{2} \int_{\Omega}\left(\overline{\mathbf{H}}_{\mathrm{eff}}^{n+1}+\overline{\mathbf{H}}_{\mathrm{eff}}^{n}\right) \cdot\left(\mathbf{m}^{n+1}-\mathbf{m}^{n}\right) \mathrm{d} \mathbf{x}\right| \\
& \quad \leq C k^{2}\left(\left\|\mathbf{v}^{n}\right\|_{L^{2}}\left\|\mathbf{v}^{n}\right\|_{L^{4}}+\left\|\mathbf{v}^{n}\right\|_{L^{2}}^{2}\right)
\end{aligned}
$$

The stray-field contribution can be bounded with the help of (25) with $p=4$. The contributions in the second line on the r.h.s. of (49) are of higher order in $k$. The first term can be easily bounded using Young's inequality:

$$
\left|\int_{\Omega} \frac{\partial \overline{\mathbf{H}}_{\mathrm{eff}}}{\partial \mathbf{m}}\left(\mathbf{v}^{n}\right) \cdot \mathbf{v}^{n} \mathrm{~d} \mathbf{x}\right| \leq C\left\|\mathbf{v}^{n}\right\|_{L^{2}}^{2} .
$$

Plugging in (50) and (51) into (49) yields that

$$
\begin{aligned}
& \mathcal{E}\left(\mathbf{m}^{n+1}\right)-\mathcal{E}\left(\mathbf{m}^{n}\right)+k \int_{\Omega} \varphi\left(\mathbf{m}^{n}\right)\left|\mathbf{v}^{n}\right|^{2} \mathrm{~d} \mathbf{x} \\
& \quad \leq C k^{2}\left(\left\|\mathbf{v}^{n}\right\|_{L^{2}}+\left\|\mathbf{v}^{n}\right\|_{L^{2}}^{2}+\left\|\mathbf{v}^{n}\right\|_{L^{2}}\left\|\mathbf{v}^{n}\right\|_{L^{4}}+\rho(k) d^{2}\left\|\nabla \mathbf{v}^{n}\right\|_{L^{2}}^{2}\right) \\
& \quad \leq C^{\prime} k^{2}\left(\left\|\mathbf{v}^{n}\right\|_{L^{2}}+\left\|\mathbf{v}^{n}\right\|_{L^{2}}^{2}+\left\|\mathbf{v}^{n}\right\|_{L^{2}}\left\|\nabla \mathbf{v}^{n}\right\|_{L^{2}}+\rho(k)\left\|\nabla \mathbf{v}^{n}\right\|_{L^{2}}^{2}\right),
\end{aligned}
$$


where $C$ denotes a generic constant. Here we made use of the classical Sobolev embedding

$$
\left\|\mathbf{v}^{n}\right\|_{L^{4}} \leq C\left\|\nabla \mathbf{v}^{n}\right\|_{L^{2}}
$$

Summing from $n=0$ to $N-1$ leads to

$$
\begin{aligned}
& \mathcal{E}(\mathbf{m}(N k))-\mathcal{E}(\mathbf{m}(0))+\int_{\Omega_{T}} \varphi_{M}\left(\mathbf{m}_{h, k}^{-}\right)\left|\mathbf{v}_{h, k}\right|^{2} \mathrm{~d} \mathbf{x} \mathrm{d} t \\
& \quad \leq C k\left(\left\|\mathbf{v}_{h, k}\right\|_{L^{2}}+\left\|\mathbf{v}_{h, k}\right\|_{L^{2}}^{2}+\left\|\mathbf{v}_{h, k}\right\|_{L^{2}}\left\|\nabla \mathbf{v}_{h, k}\right\|_{L^{2}}+\rho(k)\left\|\nabla \mathbf{v}_{h, k}\right\|_{L^{2}}^{2}\right) .
\end{aligned}
$$

We are now ready to pass to the limit. Noticing once again that $k\left\|\nabla \mathbf{v}^{n}\right\|_{L^{2}\left(\Omega_{T}\right)}$ is uniformly bounded from (39) we derive that

$$
\mathcal{E}(\mathbf{m}(N k))-\mathcal{E}(\mathbf{m}(0))+\alpha \int_{\Omega_{T}}\left|\mathbf{v}^{n}\right|^{2} \mathrm{~d} \mathbf{x} \mathrm{d} t \leq 0 .
$$

\section{Numerical results}

In this section, we report on two numerical experiments designed to test the scheme. The first one is intended to confirm the order of the scheme (expected to be nearly 2 ); the second one investigates the effect of overdamping i.e. taking $\rho$ as suggested in Theorem 2. In both cases, we refer to numerical test cases that are well-known in the physics literature of ferromagnetic materials (see for instance [12]).

\subsection{Testing the order of the scheme}

In submicron Permalloy dots, the two main contributions to the effective field $\mathbf{H}_{\text {eff }}(\mathbf{m})$ are the exchange field $\mathbf{H}_{\mathrm{ex}}(\mathbf{m})$ and the demagnetizing field $\mathbf{H}_{\mathrm{d}}(\mathbf{m})$, solution to (4). In our finite element code "feeLLGood", $\mathbf{H}_{\mathrm{d}}(\mathbf{m})$ is computed by the FMM [14].

The first study mentioned above addresses the dynamical relaxation of the magnetization towards equilibrium in a flat cylindrical dot which is $200 \mathrm{~nm}$ wide and $20 \mathrm{~nm}$ thick. Typical material parameters are $A=1.0 \times 10^{-11} \mathrm{Jm}^{-1}$ and $M_{S}=$ $8.0 \times 10^{5} \mathrm{Am}^{-1}$ corresponding to an exchange length

$$
d=\left(2 A \mu_{0}^{-1} M_{s}^{-2}\right)^{1 / 2}=5 \mathrm{~nm}
$$

The damping factor $\alpha$ in the Landau-Lifschitz equation is set to 0.05 , and we have taken $\gamma_{0}=2.21 \times 10^{5} \mathrm{~mA}^{-1} \mathrm{~s}^{-1}$ [(cf. (1)].

The initial state is a vortex whose core is displaced in the $x$-direction. Writing in complex variables and renormalizing the radius of the dot to 1 , the initial magnetization 
Fig. 2 Initial vortex distribution in a unit disk with $a=c=0.5$

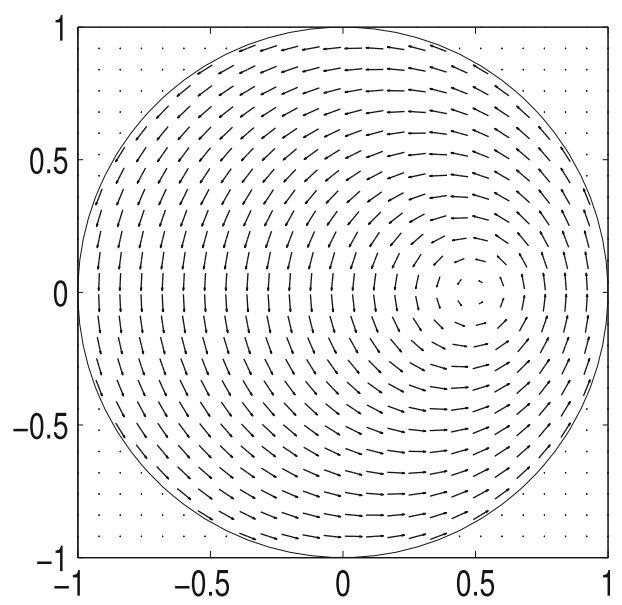

distribution is then given by

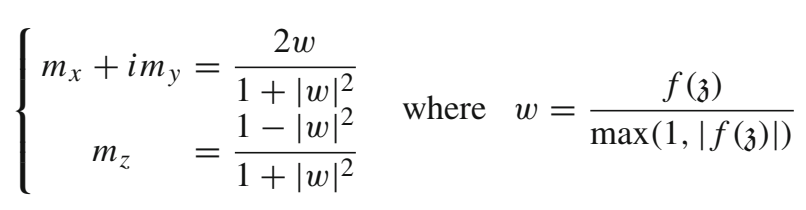

with the complex function $f(\mathfrak{z})=\frac{i}{c} \frac{\mathfrak{z}-a}{1-a \mathfrak{z}}$ and $\mathfrak{z}=x+i y$. Parameters $a \in[0,1)$ and $c \in \mathbb{R}$ respectively specify the displacement of the vortex core from the sample's center and the characteristic size of the vortex. Our simulation uses $a=c=0.5$. The corresponding initial magnetization distribution is depicted in Fig. 2.

Notice that our construction ensures a distribution locally tangent to the circular boundary. It is well known that the stray-field energy strongly penalizes non-tangential configurations in thin films and thus, at least over short times, the nucleation of further vortices. The simulations were performed on a tetrahedral mesh of 46,016 elements $(8,355$ nodes $)$ with an in-plane mesh-size $h=4 \mathrm{~nm}<d$. The final simulation time is $T=10$ ns, while the bound $M\left[\right.$ (cf. (20)] is set s.t. $k M(2 \alpha)^{-1}=0.1$.

Figure 3 shows the time evolution of the average magnetization components and monitors the convergence of the scheme as the time step tends to 0. At large time the vortex turns as expected around the dot's center at a characteristic frequency of $f=$ $0.62 \mathrm{GHz}$ in agreement with the analytical value given by Guslienko's ferromagnetic resonance model [12] within a physically acceptable error of about $10 \%$.

During its first rotation, the vortex core dilates, dissipating excess energypredominantly exchange energy. The rotation and core expansion combined altogether cause fast magnetization variations, as observed in Fig. 3 at short times.

We performed simulations with a range of time steps to study the order of the scheme. A log-log plot of the energy gap of the solution at the intermediate time $t=0.7 \mathrm{~ns}$ and an estimation corresponding to $k \rightarrow 0$ is presented in Fig. 4. The gap follows a quadratic power law, confirming the presumed quadratic order. 

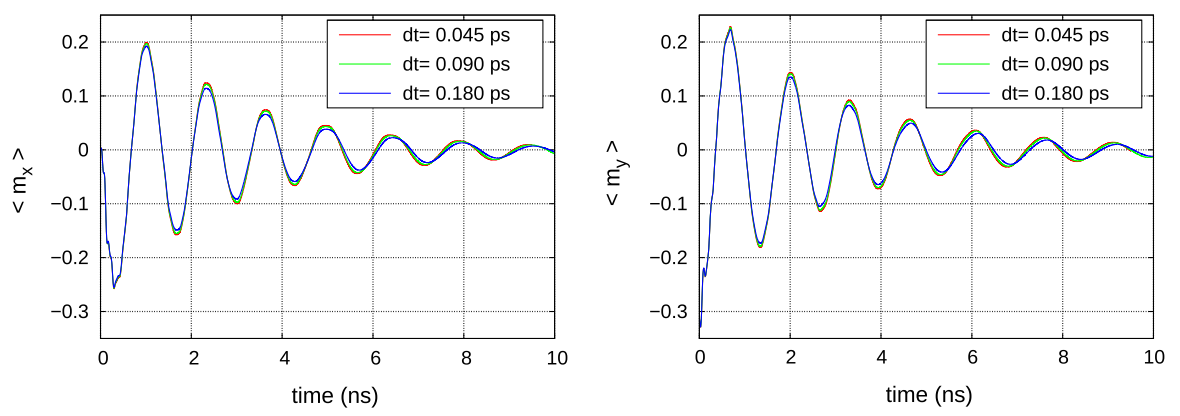

Fig. 3 Temporal evolution of the average magnetization

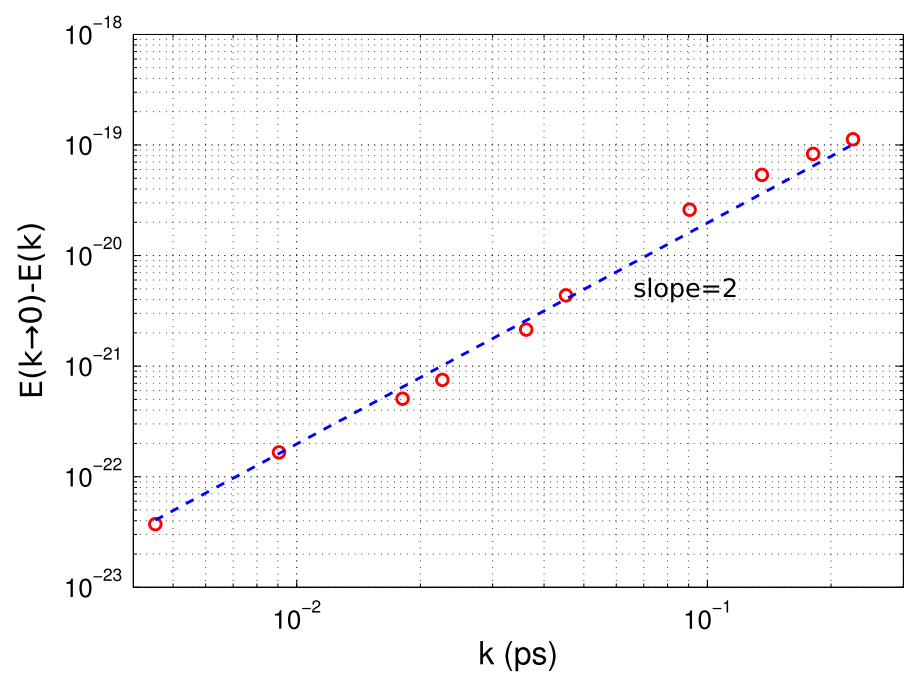

Fig. 4 Energy gap versus time step following almost a quadratic law

\subsection{Influence of $\rho$ on stability}

We now consider a Permalloy dot with an elliptic $120 \mathrm{~nm} \times 75 \mathrm{~nm}$ cross section and $2.5 \mathrm{~nm}$ thickness. It is discretized into 23,862 tetrahedral elements (4,732 nodes). The material parameters are the same as in the preceding experiment.

Starting from a saturated magnetization state along the [110] direction, the system relaxes to its equilibrium configuration: a symmetric distribution roughly aligned with the long axis of the ellipse. Again, the magnetization tends to be tangent to the ellipse boundary, in order to minimize the demagnetizing energy.

In order to test the effect of the (slight) overdamping induced by the parameter $\rho$, we performed two simulations with the same time step $k=1$ ps but with different values for $\rho$ :

1. $\rho=0$, and

2. $\rho(k)=\frac{k}{\tau}\left|\ln \frac{k}{\tau}\right| \approx 0.022$, corresponding to the characteristic time $\tau=250$ ps. 


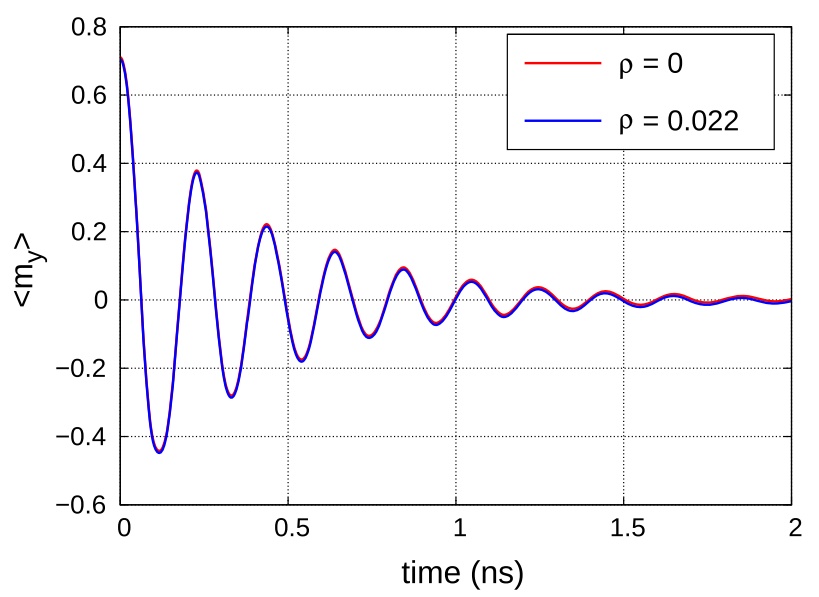

Fig. 5 Time evolution of the average magnetization component along Oy with $k=1$ ps obtained with $\rho=0$ and $\rho \approx 0.022$
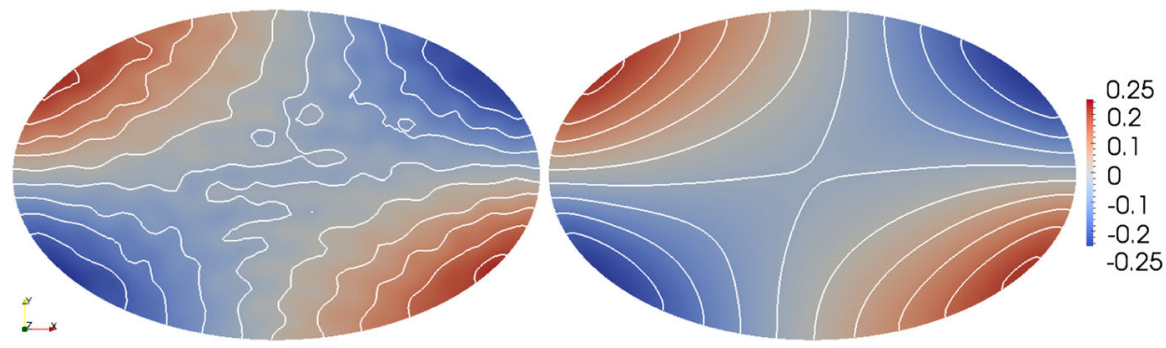

Fig. 6 Distribution of the $y$-component of the magnetization along the $y$-axis at $t=1 \mathrm{~ns}$ for $\rho=0($ left $)$ and $\rho \approx 0.022$ (right)

In Fig. 5, the evolution of the average magnetization seems almost identical ( $2 \mathrm{~ns})$, independent of $\rho$. A look at Fig. 6, however, reveals that the magnetization distributions differ significantly, with an instability occurring in the case $\rho=0$.

In Fig. 7, which shows the evolution of the energy, one clearly observes the stabilizing effect of $\rho$ in the numerical scheme. This effect was observed in practice whatever the time step used. For $\rho=0$ it is necessary to drop down the time step (typically below $k_{c} \approx 0.5 \mathrm{ps}$ in our case) to recover stability.

\section{Summary}

We started out with an introduction and discussion of the newly proposed temporal scheme. We proved its order 2 consistency to the equation, its uniform stability and (weak) convergence. Numerical simulations on physical test cases show strong evidence of good performance and confirm in practice the theoretical properties of the algorithm stated in Theorem 2, namely the quadratic convergence and stabilizing effect of $\rho$. A rigorous proof of the order 2 convergence is yet missing, though, due 


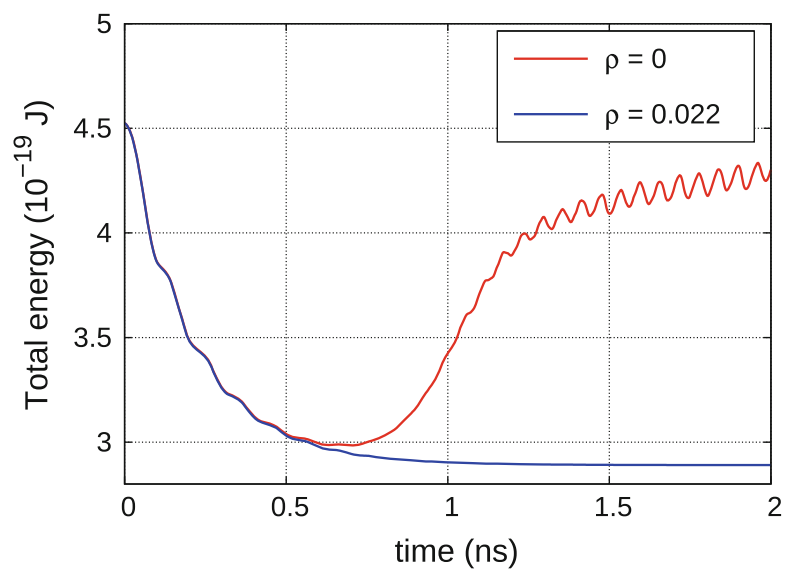

Fig. 7 Total energy versus time for $\rho=0$ (red) and $\rho \approx 0.022$ (blue)

to the very strong nonlinearities in the equations such a result is clearly out of reach for the time being.

Acknowledgments FA and JS both acknowledge support from the chair "Mathematical Modelling and Numerical Simulation, F-EADS-Ecole Polytechnique-INRIA-F-X" and the ANR project ANR-08-BLAN0199. JS acknowledges support from the German Science Foundation through the Collaborative Research Center 611 "Singular Phenomena and Scaling in Mathematical Models".

\section{References}

1. Alouges, F.: A new finite element scheme for Landau-Lifschitz equations. Disc. Cont. Dyn. Syst. Ser. S 1(2), 187-196 (2008)

2. Alouges, F.: A new algorithm for computing liquid crystal stable configurations: the harmonic mapping case. SIAM J. Numer. Anal. 34(5), 1708-1726 (1997)

3. Alouges, F., Kritsikis, E., Toussaint, J.-C.: A convergent finite element approximation for the LandauLifschitz-Gilbert equation. Physica B 407 (2012)

4. Alouges, F., Conti, S., DeSimone, A., Pokern, Y.: Energetics and switching of quasi-uniform states in small ferromagnetic particles. M2AN Math. Model. Numer. Anal. 38(2), 235-248 (2004)

5. Alouges, F., Jaisson, P.: Convergence of a finite element discretization for the Landau-Lifshitz equations in micromagnetism. Math. Model. Method. Appl. Sci. 16(2), 299-316 (2006)

6. Alouges, F., Soyeur, A.: On global weak solutions for Landau-Lifchitz equations: existence and nonuniqueness. In: Nonlinear analysis, theory, methods and applications 18(11), 1071-1084 (1992)

7. Bartels, S.: Stability and convergence of finite-element approximation schemes for harmonic maps. SIAM J. Numer. Anal. 43(1), 220-238 (2005)

8. Bartels, S., Ko, J., Prohl, A.: Numerical analysis of an explicit approximation for the Landau-LifshitzGilbert equation. Math. Comp. 77, 773-788 (2008)

9. Bartels, S., Prohl, A.: Convergence of an implicit finite element method for the Landau-Lifshitz-Gilbert equation. SIAM J. Numer. Anal. 44(4), 1405-1419 (2006)

10. Brown, W.F.: Micromagnetics. Wiley, New York (1963)

11. Cimràk, I.: A survey on the numerics and computations for the Landau-Lifshitz equation of micromagnetism, pp. 277-309. ACME, Netherlands (2007)

12. Guslienko et al: Eigenfrequencies of vortex state excitations in magnetic submicron-size disks. J. Appl. Phys. 91, 8037-8040 (2002)

13. Hubert, A., Schäfer, R.: Magnetic domains. Springer, Berlin (1998) 
14. Kritsikis, E., Toussaint, J.-C., Fruchart, O., Szambolics, H., Buda-Prejbeanu, L.: Fast computations of magnetostatic fields by non-uniform fast Fourier transforms. Appl. Phys. Lett. 93, 132508 (2008)

15. Landau, L., Lifschitz, I.: On the theory of the dispersion of magnetic permeability in ferromagnetic bodies. Phys. Zeitsch. der Sow. 8, 153-169 (1935)

16. Szambolics, H., Toussaint, J.-C., Buda-Prejbeanu, L., Alouges, F., Kritsikis, E., Fruchart, O.: Innovative weak formulation for the LLG equation. IEEE Trans. Magn. 44(11), 3153-3156 (2008)

17. Vanselow, R.: About Delaunay triangulations and discrete maximum principles for the linear conforming FEM applied to the Poisson equation. Appl. Math. 46(1), 13-28 (2001)

18. Visintin, A.: On Landau-Lishitz equations for ferromagnetism. Jpn. J. Appl. Math. 2, 69-84 (1985) 